\title{
VISCOUS MOTION IN AN OCEANIC CIRCULATION MODEL
}

\author{
A.F. Bennett and P.E. Kloeden
}

\begin{abstract}
The barotropic motion of a viscous fluid in a laboratory simulation of ocean circulation may be modelled by Beardsley's vorticity equations. It is established here that these equations have unique smooth solutions which depend continuously on initial conditions. To avoid a boundary condition which involves an integral operator, the vorticity equations are replaced by an equivalent system of momentum equations. The system resembles the two-dimensional incompressible Navier-stokes equations in a rotating reference frame. The existence of unique generalized solutions of the system in a square domain is established by modifying arguments used by Ladyzhenskaya for the Navier-Stokes equations. Smoothness of the solutions is then established by modifying Golovkin's arguments, again originally for the NavierStokes equations. A numerical procedure for solving the vorticity equations is discussed, as are the effects of reentrant corners in the domain modelling islands and peninsulae.
\end{abstract}

\section{Introduction}

Laboratory experiments with rotating fluids have led to an improved understanding of the physics of large scale ocean circulation. They also provide ideal tests for numerical models of ocean circulation. These more flexible numerical models can then be used to study the physical effects

Received 18 December 1980. The paper was prepared while Dr Kloeden was on leave from the School of Mathematical and Physical Sciences, Murdoch University, Murdoch, Western Australia. 
of, for example, density stratification and variable wind stress, neither of which is easily simulated in the laboratory.

The first author has developed a sliced box laboratory experiment to simulate large scale barotropic motion in the ocean. This motion can be modelled with a system of partial differential equations due to Beardsley $[3,4,5]$, which is an informal asymptotic approximation to the incompressible Navier-Stokes equations in a rotating reference frame. These equations are presently being solved numerically by Holland with the methods developed in his eddy-resolving general circulation model [8]. Although the asymptotic expansion used to derive the equations is not uniformly valid, there is good agreement between the numerical calculations and observations. These results will be presented elsewhere by Holland and Bennett [9]. The purpose of this paper is to prove that Beardsley's equations have solutions which are smooth enough to ensure the consistency of the centred finite difference techniques used in these numerical calculations (the convergence of the numerical procedure is not considered here).

The laboratory experiment developed by the first author is described briefly in Section 2, and Beardsley's equations are presented in Section 3. These involve a parabolic partial differential equation for the (vertical component of) vorticity, weakly coupled to a Poisson equation for the streamfunction, together with appropriate initial conditions and boundary conditions. The latter correspond to a no-slip boundary condition for the depth-independent horizontal velocity. In Section 4 the numerical procedure being used by Holland is briefly described, and the smoothness of the vorticity and streamfunction sufficient to ensure its consistency is determined. Then in Section 5 Beardsley's equations are replaced by an equivalent system of momentum equations which closely resemble the twodimensional incompressible Navier-Stokes equations in a rotating reference frame. This is done because the boundary condition for the parabolic vorficity equation involves an integral operator and, as far as we are aware, the theory of heat potentials has not yet been extended to such boundary conditions. On the other hand, the existence of generalized solutions for the equivalent system of momentum equations is readily established by modifying Ladyzhenskaya's proof [11, Chapter 6] of the existence of such generalized solutions for the two-dimensional 
incompressible Navier-Stokes equations. This is carried out in Section 6 , and then in Section 7 these generalized solutions are shown to in fact be clessical solutions with the appropriate smoothness required for the consistency of the numerical scheme discussed in Section 4. This is done by modifying Golovkin's proof [6], for the Navier-Stokes equations on a domain with a smooth boundary, to the momentum equations under consideration on a square domain. In Section 8 the effect on the smoothness of solutions is discussed when a barrier or reentrant corner, modelling an island, is included in the region of motion.

\section{The experimental apparatus}

The experimental apparatus is a glass box, which is filled with water and mounted on a turntable. The box has square horizontal cross-section and a sloping top. It has width $L$, maximum depth $H$ and top slope $\alpha$ (with respect to the horizontal). The sides and top of the box are fixed to the turntable, whereas the bottom of the box is a disc which can move independently of the turntable. A water jacket surrounds the lower part of the box to prevent water from escaping through the $0.1 \mathrm{~mm}$ gap separating the disc from the sides of the box. See Figure 1, page 446.

The turntable rotates about the laboratory vertical at a rate $\Omega$ relative to the laboratory, while the disc rotates about the laboratory vertical at a rate $\omega$ relative to the turntable (hence at a rate $\Omega+\omega$ relative to the laboratory). The rotation of the disc produces motion in the water relative to the rotating reference frame of the turntable. The effect of the sloping top of the box is to stretch columns of water which are parallel to the axis of rotation. This simulates the effect of the latitudinal variation in the vertical component of the earth's rotation rate, that is the B-effect [13]. See Figure 2, page 447.

The flow in the box is made visible by an opalescent paint suspension in strong side lighting, and is observed through the sides and top of the box. 


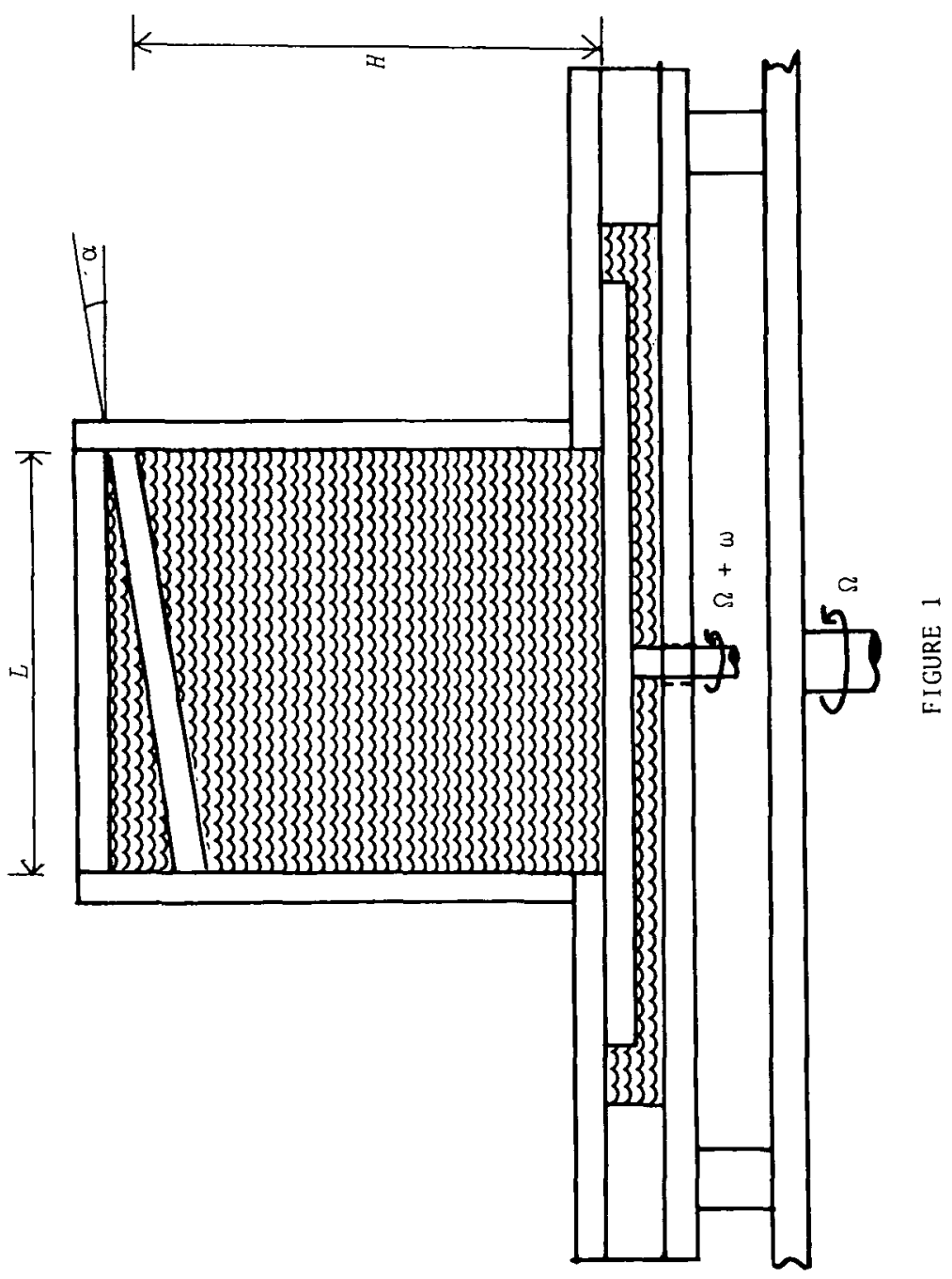




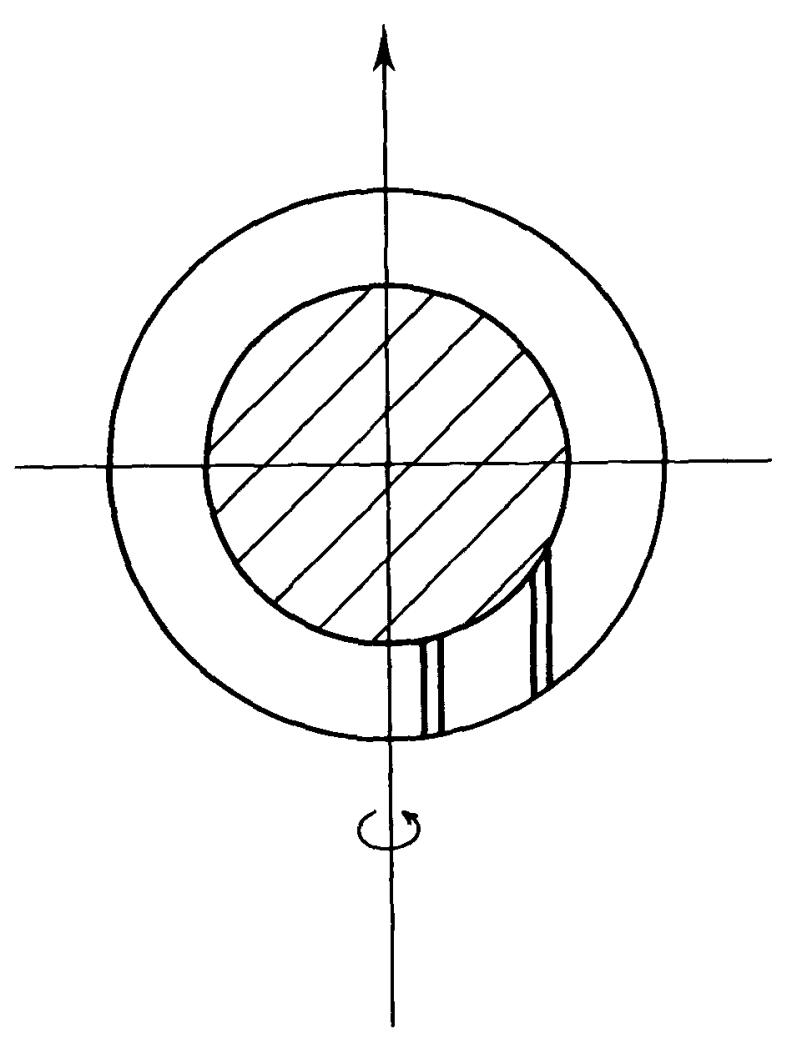

FIGURE 2

\section{The vorticity equations}

The motion of the water in the box can be modelled by an informal approximation to the incompressible Navier-Stokes equations in the parameter range $\alpha \ll 1$, Ro $\ll 1$ and $E \ll 1$, where Ro $=|\omega| /|\Omega|$ is the Rossby number, $\mathbf{E}=\nu /|\Omega| H^{2}$ the Ekman number and $\nu$ the kinematic viscosity of water. The horizontal velocity here is independent of depth, that is, it is barotropic [14].

Dimensionless variables are used, with $t \geq 0$ denoting time; 
$\mathrm{X}=(x, y) \in S=(0,1) \times(0,1)$ the horizontal cartesian coordinates (with $x$ increasing to the east and $y$ to the north); $u=(u, v)$ the horizontal velocity; $\psi$ the stream function;

$$
\zeta=\frac{\partial v}{\partial x}-\frac{\partial u}{\partial y}
$$

the (vertical component of) vorticity; and

$$
d=d(y)=1-(1-y) \frac{\tan \alpha}{\lambda}
$$

the depth of the box, where $\lambda=H / L$ is the aspect ratio. The corresponding dimensional variables here are respectively $t /|\omega|, L x$, $|\omega| L u,|\omega| L^{2} \psi,|\omega| \zeta$ and $H d$. Let $\sigma=\operatorname{sgn} \Omega, \delta=\operatorname{sgn} \omega$ and let $\nabla^{2}$ be the horizontal Laplacian operator. Then following Beardsley $[3,4,5]$ the vorticity equations are

$$
\frac{\partial \zeta}{\partial t}+u \frac{\partial \zeta}{\partial x}+v \frac{\partial \zeta}{\partial y}-\frac{2 \sigma \tan \alpha}{d \mathrm{Ro}} v=-\frac{2 \mathrm{E}^{\frac{3}{2}}}{\partial \mathrm{Ro}} \zeta+\frac{\lambda^{2} \mathrm{E}}{\mathrm{Ro}} \nabla^{2} \zeta+\frac{2 \sigma \mathrm{E}^{\frac{3}{2}}}{d \mathrm{Ro} O},
$$

$$
\nabla^{2} \psi=\zeta
$$

and

$$
u=-\frac{\partial \psi}{\partial y}, \quad v=\frac{\partial \psi}{\partial x} .
$$

The third term on the right hand side of equation (3.3) represents the source of vorticity produced by the spinning disc. It should be noted that equations (3.3)-(3.5) cannot be derived as a formal asymptotic expansion of the Navier-Stokes equations which is uniformly valid for all $x$ in $S$ as $\alpha$, Ro and $E+0$. This is because the first term on the right side of equation (3.3) dominates the second term near the shallow and deep ends of the bix (that is, in the southern and northern boundary layers), while the second dominates the first near the other ends of the box (that is, in the western and eastern boundary layers).

The non-slip boundary condition (that is, $u=0$ on $\partial S$ ) implies that the streamfunction satisfies

$$
\psi=0 \text { on } \partial S \text { for all } t \geq 0
$$

which provides a Dirichlet boundary condition for the Poisson equation (3.4). No-slip also implies that 


$$
\frac{\partial \psi}{\partial n}(x, t)=\iint \frac{\partial G}{\partial n}\left(x, x^{\prime}\right) \zeta\left(x^{\prime}, t\right) d x^{\prime}=0
$$

on $\partial S$ for all $t \geq 0$, where $G\left(x, x^{\prime}\right)$ is the Green's function for the Dirichlet problem (3.4) and (3.5). Equation (3.7) gives the boundary condition for equation (3.3), which being a parabolic equation also requires an initial condition

$$
\zeta(\mathrm{x}, 0)=\zeta_{I}(\mathrm{x}) \text { for } x \in S \text { at } t=0 .
$$

\section{The method of numerical solution}

The numerical procedure being used by Holland to solve the equations (3.3)-(3.8) is essentially that contained in his eddy-resolving general circulation model [8]. This is most conveniently applied on a rectangular domain, which is the main reason for using a box with square horizontal cross-section in the experimental apparatus, rather than a circular cylinder as used by, for example, Beardsley $[3,4,5]$.

Holland's procedure requires the three equations (3.3)-(3.5) to be replaced by a single evolution equation involving the streamfunction $\psi$, namely

(4.1) $\nabla^{2}\left(\frac{\partial \psi}{\partial t}\right)=J\left(\nabla^{2} \psi, \psi\right)+\frac{2 \sigma \tan \alpha}{d \operatorname{Ro}} \frac{\partial \psi}{\partial x}-\frac{2 \mathrm{E}^{\frac{1}{2}}}{d R \circ} \nabla^{2} \psi+\frac{\lambda^{2} \mathrm{E}}{\mathrm{Ro}} \nabla^{2}\left(\nabla^{2} \psi\right)+\frac{2 \delta \mathrm{E}^{\frac{1}{2}}}{d \mathrm{Ro}}$, where $J$ is the two-dimensional Jacobian defined by

$$
J(a, b)=\frac{\partial a}{\partial x} \frac{\partial b}{\partial y}-\frac{\partial a}{\partial y} \frac{\partial b}{\partial x} .
$$

The time derivative $\frac{\partial \psi}{\partial t}$ at $(x, t)$ in $(4.1)$ is then approximated by the central difference

$$
(\psi(x, t+\Delta t)-\psi(x, t-\Delta t)) / 2 \Delta t
$$

where $\Delta t$ is the numerical time step, and the right side of equation (4.1) is evaluated (except for the biharmonic term, which is evaluated at time $(t-\Delta t)$ ) at time $t$. This leads to a Poisson problem for $\psi(x, t+\Delta t)$ with the Dirichlet boundary condition (3.6), which is discretized by means of a five-point difference scheme for the Laplacian and a nine-point Arakawa difference scheme for the Jacobian (the latter conserves discrete analogues of vorticity, total enstrophy and total kinetic energy in 
unforced, nondissipative systems [2]). This discrete Poisson problem is then solved by an exact direct method. See [8] for further details.

The above numerical procedure clearly requires the vorticity $\zeta=\nabla^{2} \psi$ to be continuously differentiable in $t$ and twice continuously differentiable in $x$ and $y$. In the procedure the discretized Laplacian and Jacobian schemes need only be centred on interior grid points and not on boundary points. However uniform bounds on the second order spatial derivatives of $\zeta$ are required at all interior points, to ensure the consistency of the numerical procedure as the numerical step lengths $\Delta x$ and $\Delta y \rightarrow 0$. For this it is sufficient that the vorticity $\zeta$ be in $C^{2}(\bar{S})$ with $\frac{\partial \zeta}{\partial t}$ in $C^{0}(\bar{S})$, where $\bar{S}$ is the closure of $S$. The presence of the four corner points on the boundary $\partial S$ considerably complicates matters here. In the actual experimental apparatus the corners are butted glass joints rounded off with glue. The numerical procedure is however defined on a perfect square domain, so the corner points cannot be disregarded.

\section{An equivalent system of momentum equations}

When the streamfunction is known, the vorticity equation (3.3) is a linear second order parabolic partial differential equation. Initial boundary value problems for such equations can often be solved with the aid of heat potentials [10]. Here, however, the boundary condition (3.7) involves an integral operator to which, as far as we are aware, the theory of heat potentials has rot yet been extended. To circumvent this difficulty, the vorticity equations (3.3)-(3.8) will be replaced by the following system of momentum equations:

$$
\begin{aligned}
& \frac{\partial u}{\partial t}+u \frac{\partial u}{\partial x}+v \frac{\partial u}{\partial y}-2 \sigma V=-\frac{\partial p}{\partial x}+\frac{\lambda^{2} \mathrm{E}}{\mathrm{Ro}} \nabla^{2} u, \\
& \frac{\partial v}{\partial t}+u \frac{\partial v}{\partial x}+v \frac{\partial v}{\partial y}+2 \sigma U=-\frac{\partial p}{\partial y}+\frac{\lambda^{2} \mathrm{E}}{\mathrm{Ro}} \nabla^{2} v,
\end{aligned}
$$$$
\frac{\partial u}{\partial x}+\frac{\partial v}{\partial y}=0
$$$$
\frac{\partial U}{\partial x}+\frac{\partial V}{\partial y}=\frac{\sigma E^{\frac{1}{2}}}{d \operatorname{Ro}}\left(\frac{\partial v}{\partial x}-\frac{\partial u}{\partial y}-\delta\right)-\frac{\operatorname{Rotan} \alpha}{d \lambda} v,
$$ 


$$
\text { (5.5) } \quad \frac{\partial V}{\partial x}-\frac{\partial U}{\partial y}=0,
$$

with initial condition

$$
u(x, 0)=u_{I}(x)=\left(u_{I}(x), v_{I}(x)\right) \text { for } x \in S \text { at } t=0,
$$

and boundary conditions

$$
u(x, t)=0 \text { for } x \in \partial S, t \geq 0
$$

and

$$
U \cdot \hat{n}=0 \text { on } \partial S \text { for } t \geq 0
$$

where $U=(U, V)$ and $\hat{n}$ is the normal to $\partial S$.

Readers familiar with the quasigeostrophic approximation [14] will recognize equations $(5.1)$ and $(5.2)$ as the $O($ Ro) momentum equations with $u=(u, v)$ as the $O(1)$ horizontal velocity and $U=(U, V)$ as the irrotational part of the $O(R O)$ velocity, the solenoidal part of which can be represented by a streamfunction and hence absorbed into the pressure gradient terms. Note that the non-uniqueness of the decomposition of the $O(R o)$ velocity into irrotational and solenoidal parts permits the imposition of the rigid boundary condition (5.8) on the irrotational part (and also on the solenoidal part, although the latter is not needed here).

It is easily shown that if $u=(u, v)$ is a solution of equations (5.1)-(5.8) then its vorticity $\zeta$, defined by (3.1), is a solution of equations (3.3)-(3.8) provided that initially

$$
\zeta_{I}=\frac{\partial v_{I}}{\partial x}-\frac{\partial u_{I}}{\partial y} \text {. }
$$

\section{Existence of generalized solutions}

The momentum equations (5.1)-(5.8) closely resemble the twodimensional incompressible Navier-Stokes equations. Indeed if $\sigma=\operatorname{sgn} \Omega$ is set equal to zero in equations (5.1) and (5.2), then the initial boundary value problem $(5 . x)-(5.3),(5.6)$ and (5.7) is almost precisely the nonlinear nonstationary problem for the Navier-Stokes equations considered by Ladyzhenskaya [11], namely: 


$$
\begin{aligned}
\frac{\partial u}{\partial t}+(u \cdot \nabla) u & =-\nabla p+\frac{1}{\operatorname{Re}} \nabla^{2} u+F, \\
\nabla \cdot u & =0, \\
u(x, 0) & =u_{I}(x) \text { for } x \in S \text { at } t=0
\end{aligned}
$$

and

$$
u(x, t)=0 \text { for } x \in \partial S \text { and } t \geq 0 \text {, }
$$

where $R e$ is the Reynolds number (equal to $R \circ / \lambda^{2} E$ here), $F$ is a specified body force, and $\boldsymbol{\nabla}$ is the horizontal gradient operator.

Ladyzhenskaya has established the existence, uniqueness and continuity in initial data of generalized solutions of the nonlinear non-stationary problem (6.1)-(6.4) [11, Chapter 6]. Her proof' is based on two identities [11, Chapter 6, Section 2, Equations 7 and 8], which follow from (6.1)-(6.4). These are

$$
\frac{1}{2} \frac{d}{d t}\|u\|^{2}=-\frac{1}{\operatorname{Re}}\left\{\left\|\frac{\partial u}{\partial x}\right\|^{2}+\left\|\frac{\partial u}{\partial y}\right\|^{2}\right\}+(F, u)
$$

and

$$
\text { (6.6) } \begin{aligned}
\frac{1}{2} \frac{d}{d t}\left\|\frac{\partial u}{\partial t}\right\|^{2}=-\frac{1}{R e}\left\{\left\|\frac{\partial^{2} u}{\partial t \partial x}\right\|^{2}+\left\|\frac{\partial^{2} u}{\partial t \partial y}\right\|^{2}\right\}+\left(\frac{\partial F}{\partial t}, \frac{\partial u}{\partial t}\right) \\
-\iint_{S}\left\{\frac{\partial u}{\partial t} \frac{\partial u}{\partial x} \cdot \frac{\partial u}{\partial t}+\frac{\partial v}{\partial t} \frac{\partial u}{\partial y} \cdot \frac{\partial u}{\partial t}\right\} d x,
\end{aligned}
$$

where the inner product is defined by

$$
(a, b)=\iint_{S} a \cdot b d x
$$

and the norm by

$$
\|a\|=(a, a)^{\frac{3}{2}} .
$$

Identities analogous to $(6.5)$ and $(6.6)$ can be derived for the momentum equations $(5.1)-(5.8)$. The first of these is

(6.9) $\frac{d}{d t}\|u\|^{2}=-\frac{1}{\operatorname{Re}}\left\{\left\|\frac{\partial u}{\partial x}\right\|^{2}+\left\|\frac{\partial u}{\partial y}\right\|^{2}\right\}+(F, u)$

$$
-\frac{2 \mathrm{E}^{\frac{1}{2}}}{\mathrm{Ro}} \iint_{S} \frac{1}{d}\left\{|\nabla \psi|^{2}-\frac{3}{2}\left(\frac{\tan \alpha}{\lambda d}\right)^{2} \psi\right\} d \mathrm{x}
$$


and the second, which is similar, is omitted. Here $\psi$ is the streamfunction corresponding to the horizontal velocity $u$ and $F=\left(F_{1}, F_{2}\right)$ is an effective body force with components

$$
F_{1}(x, y)=-\frac{\delta \lambda E^{\frac{1}{2}}}{\operatorname{Rotan} \alpha} \ln d(y)
$$

and

$$
F_{2}(x, y)=\frac{\delta E^{\frac{1}{2}}}{\text { Ro }} \frac{x}{d(y)} .
$$

Note that the box depth $d(y)$, which is defined by (3.2), satisfies

$$
0<1-\frac{\tan \alpha}{\lambda}=d(0) \leq d(y) \leq d(1)=1 \text {. }
$$

Hence the effective body force $F$ is in $C^{\infty}(\bar{S})$

Ladyzhenskaya used identities (6.5) and (6.6) to derive a priomi estimates of the form

$$
\|u\| \leq\left\|u_{I}\right\|+\int_{0}^{t}\|\mathrm{~F}\| d t .
$$

The same estimates can also be derived here, because the integral term on the right side of (6.9) is always the negative of a positive quantity. This follows from (6.12) and Wirtinger's inequality $[7$, p. 184], which yield

(6.14) $\iint_{S} \frac{1}{d}\left\{\left(\frac{\partial \psi}{\partial x}\right)^{2}-\frac{2}{2}\left(\frac{\tan \alpha}{\lambda d}\right)^{2} \psi^{2}\right\} d x \geq \iint_{S} \frac{1}{d}\left\{\left(\frac{\partial \psi}{\partial x}\right)^{2}-\psi^{2}\right\} d x$

$$
\begin{aligned}
& =\int_{0}^{1} \frac{1}{d(y)}\left\{\int_{0}^{1}\left\{\left(\frac{\partial \psi}{\partial x}\right)^{2}-\psi^{2}\right\} d x\right\} d y \\
& \geq\left(1-\frac{1}{\pi^{2}}\right) \int_{0}^{1} \frac{1}{d(y)}\left\{\int_{0}^{1}\left(\frac{\partial \psi}{\partial x}\right)^{2} d x\right\} d y
\end{aligned}
$$

Consequently the extra terms in the momentum equations $(5.1)-(5.8)$ serve only to reinforce the a priori estimates in [11].

Ladyzhenskaya used these a priori estimates to prove the existence of generalized solutions for equations (6.1)-(6.4). Her proof, which is based on a Galerkin construction, carries over almost verbatim to the momentum 
equations (5.1)-(5.8). For the latter equations a generalized solution on an arbitrary time interval $[0, T]$ is a function $u=(u, v)$ such that

$$
\iint_{S}\left(u^{4}+v^{4}\right) d x \leq C_{T} \text { uniformly in } t \in[0, T] \text {, }
$$

$$
\frac{\partial u}{\partial x}, \frac{\partial u}{\partial y}, \frac{\partial u}{\partial t} \in L_{2}(S \times[0, T]),
$$

with

(6.17) $\nabla \cdot u=0, u=0$ on $\partial S \times[0, T]$ and $u=u_{I}$ at $t=0$,

and which satisfies the partial differential equations (5.1) and (5.8) in the generalized sense that the integral

(6.18) $\int_{0}^{T} \iint_{S}\left\{\frac{\partial u}{\partial t} \cdot X+\frac{1}{R e}\left(\frac{\partial u}{\partial x} \cdot \frac{\partial X}{\partial x}+\frac{\partial u}{\partial y} \cdot \frac{\partial X}{\partial y}\right)-u u \cdot \frac{\partial X}{\partial x}-v u \cdot \frac{\partial X}{\partial y}-F \cdot X\right.$

$$
\left.-\frac{2}{d \operatorname{RoE}^{\frac{1}{2}}} u \cdot x-\frac{2}{d \operatorname{RoE}^{\frac{1}{2}}} \frac{\partial}{\partial y}\left(\frac{1}{d(y)}\right) \Phi u+\frac{2 \sigma}{\operatorname{Ro}} \frac{\partial}{\partial y}(\ln d(y)) \Phi v\right\} d x d t
$$

vanishes for all test functions $X$. These functions $X$ satisfy

$$
X, \frac{\partial X}{\partial x}, \frac{\partial X}{\partial y} \in L_{2}(S \times[0, T])
$$

with

$$
\nabla \cdot \mathrm{X}=0 \text { on } S \times[0, T], \mathrm{X}=0 \text { on } \partial S \times[0, T] \text {, }
$$

and correspond to a streamfunction $\Phi$, that is $X=\left(-\frac{\partial \Phi}{\partial y}, \frac{\partial \Phi}{\partial x}\right)$.

Following Ladyzhenskaya [11, page 31] let $J_{0,1}(S)$ be the completion of the space $\dot{J}(S)$ in the norm of the Sobolev space $W^{1,2}(S)$, where $\dot{J}(S)$ is the space of all $C^{\infty}$ solenoidal vector functions with compact support in $S$. Then the following existence theorem holds.

THEOREM 1. If the initial velocity $u_{I} \in W^{2,2}(S) \cap J_{0,1}(S)$, then there exists a generalized solution for the momentum equations (5.1)-(5.8) on an arbitrary finite time interval $[0, T]$.

In addition, for generalized solutions $u$ and $u^{\prime}$ corresponding to initial velocities $u_{I}$ and $u_{I}^{\prime}$ the estimate 
(6.21) $\quad\left\|u-u^{\prime}\right\| \leq\left\|u_{I}-u_{I}^{\prime}\right\| \exp \left\{\operatorname{Re}^{2}\left(2\left\|u_{I}\right\|^{2}-3\|F\|^{2} t^{2}\right)-\frac{E^{\frac{1}{2}}}{\operatorname{Ro}}\left(1-\frac{I}{\pi^{2}}\right) t\right\}$ can be derived. This yields the following theorem.

THEOREM 2. The generalized solutions of the momentum equations (5.1)-(5.8) are tnique and depend continuously on the initial velocity.

A similar estimate to (6.21) can be derived for generalized solutions corresponding to different body forces and implies the continuous dependence of solutions on the body force. Also estimate (6.21) can be replaced by the estimate

$$
\begin{aligned}
\left\|u-u^{\prime}\right\| & \leq\|u\|+\left\|u^{\prime}\right\| \\
& \leq\left\|u_{I}\right\|+\left\|u_{I}^{\prime}\right\|+2\|F\| t,
\end{aligned}
$$

which follows from (6.13) and the fact that the effective body force $F$ does not depend on $t$. This shows that the separation of generalized solutions does not increase faster than linearly in time $t$, and not exponentially as might be allowed from estimate (6.21).

The proofs of the preceding Theorems 1 and 2 differ only trivially from the corresponding proofs in [11], and so need not be given here.

Finally, note that the irrotational velocity $U=(U, V)$ can be represented by a velocity potential $\chi$ with

$$
U=\frac{\partial x}{\partial x}, \quad V=\frac{\partial x}{\partial y} .
$$

In view of the divergence equation $(5.4)$ and the no-slip boundary condition (5.8) this velocity potential $X$ satisfies the Poisson equation

$$
\nabla^{2} x=\frac{\sigma E^{\frac{3}{2}}}{d R o}(\zeta-\delta)-\frac{\text { Rotan } \alpha}{d \lambda} v
$$

with a homogeneous Neumann boundary condition. From this it follows that the irrotational velocity $U$ depends linearly on the solution $u$ of equations (5.1)-(5.8) and has the same degree of differentiability.

\section{Smoothness of solutions}

Ladyzhenskaya [11, Chapter 6, Section 4] has shown that the generalized solutions of $(6.1)-(6.4)$ are actually classical solutions. That is, all the derivatives in (6.1) and (6.2) are continuous in 
$\stackrel{\circ}{S} \times(0, T)$, while $u$ is continuous in $\bar{S} \times[0, T]$. However her proof requires that the domain has a smooth boundary, specifically $\partial S \in C^{2}$, and so would appear to be inapplicable to the square domain considered here.

A full examination of [11] shows that the smooth-boundary requirement enters after the re-arrangement of (6.1)-(6.4) into the form of the linearized stationary flow problem:

$$
\begin{aligned}
\frac{1}{\operatorname{Re}} \nabla^{2} \mathbf{u}+\nabla p & =\mathbf{G}, \\
\nabla \cdot \mathbf{u} & =0, \\
\mathbf{u} & =0 \text { on } 2 S,
\end{aligned}
$$

where for the moment only the homogeneous boundary condition (7.3) is being considered, and

$$
\mathbf{G}=\mathbf{F}-(\mathbf{u} \cdot \nabla) \mathbf{u}-\frac{\partial u}{\partial t} \text {. }
$$

Note that if $u$ is the generalized solution of $(6.1)-(6.4)$, then $G$ as defined by $(7.4)$ is in $L_{2}(S)$. Using the Leray hydrodynamic potentials [12], Ladyzhenskaya has shown that for such $G$, the unique solution $u$ of $(7.1)-(7.3)$ is in $w_{2}^{2}(S)$. Since $S$ is a strongly locally Lipschitz domain, it may be concluded [1, Theorem 5.4] that $u$, which is also the generalized solution of $(6.1)-(6.4)$, is in $c^{\lambda}(\bar{S}), 0<\lambda<1$.

It is the use of the Leray potentials which lead to the requirement that $2 S \in C^{2}$; for example the values of the double potential on $a S$ are not continuous at corners of $\partial S$. On the other hand we may dispense with the Leray potentials and the attendent smoothness requirement for $\partial S$, since Friedrich's Theorem [14, p. 177] ensures that $u \in W_{2}^{2}(S)$ provided that $G \in L_{2}(S)$ and $S$ is merely open. Note that for $(7.1)-(7.3)$ the test functions must be solenoidal, that is, in $j(s)$. Our generalized solution of (5.1)-(5.8) is a solution of (7.1)-(8.3) with respect to a much broader class of test functions (see $(6.19),(6.20)$ ) so Friedrich's Theorem certainly applies here. 
With the above change, the Ladyzhenskaya proof of smoothness of solutions of (6.1)-(6.4) is valid for our square domain. It is trivial to modify the proof. so that it applies also to the momentum equations (5.1)-(5.8). The degree of smoothness established thus far is inadequate for our purposes; for example we require $u \in c^{3}(\bar{S})$. Golovkin has shown, using the Leray potentials, that $u \in C^{l}(\bar{S})$ provided again that $\partial S \in C^{2}$ and also that $S$ is convex. The Golovkin proot carries over to the momentum equations $(5.1)-(5.8)$ on a convex domain with a smooth boundary, with trivial modifications involving the irrotational velocity $U$ (which, as been noted above, is linearly related to $u$ and has the same degree of smoothness). The proof can also be modified to hold for our square domains $S$. First, let $S_{\varepsilon}$ be the convex domain with a smooth boundary which is obtained from $S$ by rounding of $f$ the four corners with inscribed circular arcs of radius $\varepsilon$, where $0<\varepsilon<\frac{1}{2}$. The generalized solutions of the momentum equations (5.1)-(5.8) on the domain $S$ are also generalized solutions on the subdomain $S_{\varepsilon}$, but with inhomogeneous boundary values. By virtue of the Ladyzhenskaya smoothness theorem these boundary values are smooth, they may be smoothly extended [11, Chapter 1, Section 2.1] into $S_{\varepsilon}$, and so the boundary conditions may be homogenized [11, Chapter 6, section 4]. The Golovkin argument can then be used to show the generalized solutions are actually classical solutions in $S_{\varepsilon}$, belonging to $C^{l}\left(S_{\varepsilon}\right)$. Since this applies on the subdomain $S_{\varepsilon}$ for $0<\varepsilon<\frac{1}{2}$, it also holds on their union $U_{\varepsilon}$, which is just

$S \backslash\{4$ corners $\}$. It remains to consider the behaviour of the solutions at the corner points. From the no-slip condition (5.7), by the triangle inequality and the continuity of the derivatives of the solution up to the boundary of any subdomain $S_{\varepsilon}$, it is easily shown that the one-sided derivatives exist at each corner point, where in fact they all vanish.

Golovkin notes [6, Theorem VIII] that his method can be extended to show the smoothness of higher order generalized derivatives of the solution of the Navier-Stokes equations (6.1)-(6.4), provided the initial velocity and the forcing term are sufficiently smooth. The existence of such higher order derivatives follows as in Section 6 using a priori estimates derived 
from the appropriate evolution equations for them, which are obtained from the Navier-Stokes equations by differentiating the appropriate number of times. Again, the boundary conditions must be homogenized.

A similar situation applies for the momentum equations (5.1)-(5.8), provided the corner points are handled in the way discussed above. As the effective forcing term $F$, with components defined in (6.10) and (6.11), is a $C^{\infty}(\bar{S})$ function, the resulting degree of smoothness will depend on that of the initial velocity $u_{I}$. For the numerical procedure discussed in section 4 , the vorticity $\zeta$ is required to be in at least $C^{2}(\bar{S})$ with $\frac{\partial \zeta}{\partial t}$ in $C^{0}(\bar{S})$. This will certainly be the case if the initial velocity $u_{I}$ is in $C^{4}(S)$ and satisfies the obvious compatibility conditions, because then the solution $u$ will be in $C^{3}(\bar{S}) \cap C^{4}(S)$ with $\frac{\partial u}{\partial t}$ correspondingly smooth (the highest order derivatives need not be continuous up to the boundary). Actually in his numerical computations, Holland uses the initial velocity $u_{I} \equiv 0$, which is a $C^{\infty}(\bar{S})$ function. As the effective forcing term is also in $C^{\infty}(\bar{S})$, it follows by induction that the solution $u$ is in $C^{\infty}(\bar{S})$. See Golovkin [6, Theorem vIII].

\section{Coastal geometry}

The square domain of the ocean circulation model is extremely simple. Several important physical effects are thereby excluded, such as flow separation from the coast due to a bend or peninsula, and evolution of the nett circulation around an island. For both experimental and numerical reasons it is highly convenient to construct such bends, peninsulae and islands from rectangular shapes. However the numerical procedure discussed in Section 4 may be inadequate for the more complex geometries. For example, suppose that the ocean model includes a square island. Each corner of the island is a concave or reentrant corner with respect to the flow domain. The proof of existence of generalized solutions is unaffected by such corners. The Ladyzhenskaya smoothness theorem, amended as above, is also unaffected by such corners. However it only ensures $u \in C(\bar{S})$, and the sharper Golovkin theorem is no longer valid. The concave corner could be rounded (into the flow domain) to make a smooth concave bend, and 
Golovkin has noted that convexity of the flow domain does not "appear" essential for the existence of classical solutions provided the boundary is smooth (in this regard see also [11, Chapter 6, Section 4]). Nevertheless, we have been unable to adapt the method described in 7 above, to establish smoothness at a concave corner. On the other hand there are simple steady cellular velocity fields defined by truncated double Fourier series, and thus in $C^{\infty}\left(R^{2}\right)$, which are exact steady solutions of (6.1)-(6.4) for suitably chosen steady force fields, and which meet homogeneous boundary conditions on boundaries with concave corners at cell vertices.

\section{References}

[1] Robert A. Adams, Sobolev spaces (Pure and Applied Mathematics, 65. Academic Press [Harcourt Brace Jovanovich], New York, San Francisco, Iondon, 1975).

[2] Akio Arakawa, "Computational design for long-term numerical integration of the equations of fluid motion: two-dimensional incompressible flow. Part I", J. Comput. Phys. 1 (1966-1967), 119-143.

[3] R.C. Beardsley, "A numerical model of the wind-driven ocean circulation in a circular basin", Geophys. Fluid Dyn. 4 (1972-1973), 211-241.

[4] Robert C. Beardsley, "The 'sliced-cylinder' laboratory model of the wind-driven ocean circulation. Part 2: Oscillatory forcing and Rossby wave resonance", J. Fluid Mech. 69 (1975), 41-64.

[5] R.C. Beardsley and K. Robbins, "The 'sliced-cylinder' laboratory model of the wind-driven ocean circulation. Part 1: Steady forcing and topographic Rossby wave instability", J. Fluid Mech. 69 (1975), 27-40.

[6] Н.Н. Головнин [K.K. Golovkin], "О плосном двинении вязкой неснимаемой жидности" [The plane motion of a viscous incompressible fluid], Trudy Mat. Inst. Steklov. 59 (1960), 37-86. English Transl:

Amer. Math. Soc. Trans l. (2) 35 (1964), 297-350. 
[7] G.H. Hardy, J.E. Littlewood, G. Pólya, Inequalities (Cambridge University Press, Cambridge, 1934).

[8] William R. Holland, "The role of mesoscale eddies in the general circulation of the ocean - numerical experiments using a winddriven quasi-geostrophic model", J. Phys. Ocecnogr. 8 (1978), 363-392.

[9] William R. Holland and A.F. Bennett, "Numerical simulation of the long-term motion in a laboratory oceanic circulation experiment", in preparation.

[10] А.М. Нльин, А.С. Налашнинов, О.А. Олейнин [А.М. 11'in, A.S. Kalashnikov, O.A. Oleinik], "Линейные уравнения второго порядна параболнчесного типа" [Linear' equations of the second order of parabolic type], Uspehi Mat. Nauk 17 (1962), no. 3, 3-146; English Transl: Russian Math. Surveys 17 (1962), no. 3, 1-143.

[11] O.A. Ladyzhenskaya, The mathematical theory of viscous incompressible flow, Second English edition, revised and enlarged (Translated by Richard A. Silverman and John Chu. Mathematics and its Applications, 2. Gordon and Breach, New York, London, Paris, 1969).

[12] Jean Leray, "Essai sur les mouvements plans d'un liquide visqueux que limitent des parois", J. Math. Pures Appl. (9) 13 (1934), $331-418$.

[13] Joseph Pedlosky, Geophysical fluid dynamics (Springer-Verlag, New York, Heidelberg, Berlin, 1979).

[14] Kôsaku Yosida, Functional analysis, 3rd edition (Die Grundlehren der mathematischen Wissenschaften, 123. Springer-Verlag, Berlin, Heidelberg, New York, 1971).

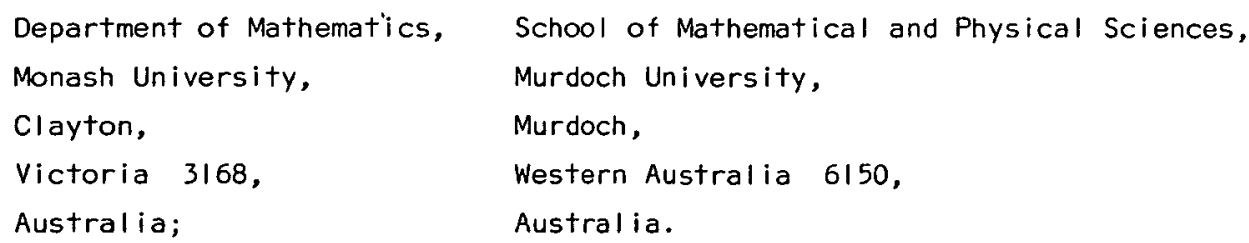

